\title{
Modernización vitivinícola del Valle de Casablanca: potencialidades y vulnerabilidad de la actividad
}

\author{
Margarita Riffo Rosas \\ Sociedad Chilena de Ciencias Geográficas. Santiago, Chile. \\ mriffo@uchilefau.cl \\ Pamela Castro Osorio \\ Departamento de Geografía, Universidad de Chile. Santiago, Chile. \\ pamcastr@uchilefau.cl

\begin{abstract}
RESUMEN
El artículo muestra algunos resultados obtenidos durante el desarrollo del Proyecto IPGH-OEA GEO 2.1.2.3.4. titulado "Expansión de la Vitivinicultura y su relación con el Desarrollo Local en Casablanca (Chile) y Mendoza (Argentina)". Se analizan las potencialidades de la comuna de Casablanca para el desarrollo de la Vitivinicultura, actividad económica que marca la especialización del valle a partir de la modernización y reconversión productiva, y que junto con la localización espacial de las agroindustrias denominadas "bodegas boutiques" se distinguen en el paisaje rural de la comuna. Se analizan también las limitantes que amenaza n la futura expansión de la vitivinicultura en el valle de Casablanca, referidas específicamente a la escasez del recurso agua en términos de precipitaciones, infraestructura de riego y sistemas de regadío, además de la competencia eventual de nuevas regiones productivas o exportadoras, lo cual representa una seria limitante para el desarrollo sustentable de la actividad.
\end{abstract}

Palabras clave: Vitivinicultura, Potencialidades, Vulnerabilidad.

\section{Modernization Casablanca Valley wine: potential and vulnerability of the activity}

\begin{abstract}
This article presents some results obtained during the development of the Project IPGH-OEA GEO 2.1.2.3 4. entitled "Expansion of the Viticulture and its relation with the Local Development in Casablanca (Chile) and Mendoza (Argentina)", whereby, the potential for the development of the Viticulture in the commune of Casablanca is analyzed. This is an economic activity that marks the specialization of the valley since its modernization and productive reconversion, and, along with the space location of agro-industries denominated "warehouses boutiques", stands out in the rural landscape of the commune. The limitations that threaten the future expansion of the viticulture in the valley of Casablanca are also analyzed, with specific reference to the shortage of water resources in terms of precipitations, irrigation infrastructure, and systems of irrigated land, in addition to the possible competition of new productive or exporting regions, which represents a serious limitation for the sustainable development of the activity.
\end{abstract}

Key words: Vineyards culture (viticulture), Potentialities, Vulnerability 


\section{INTRODUCCIÓN}

La Vitivinicultura constituye una de las actividades económicas más dinámicas de Chile, favorecida por factores endógenos derivados del aprovechamiento de ventajas comparativas de tipo agroclimático y por factores exógenos vinculados a las condiciones de los mercados internacionales en términos de demanda y precios, ocupando por ello el $5^{\circ}$ lugar entre los países exportadores de vino a escala mundial, a partir de una superficie plantada de 104.716,9 hectáreas (SERVICIO AGRÍCOLA Y GANADERO, SAG 2008).

La vitivinicultura de exportación se desarrolla en Chile entre las regiones de Atacama y Bio Bio, ocupando tanto zonas próximas al litoral como a la Cordillera de Los Andes, lo cual repercute en la variedad de cepas que distinguen los viñedos, destacando la plantación de cepas tintas en el norte y el interior del territorio, en tanto las cepas blancas se orientan preferentemente hacia la costa y la zona sur del país.

El Valle de Casablanca, situado en la provincia de Valparaíso, constituye una de las zonas vitivinícolas próxima al sector costero, destacando la producción de vinos blancos, como el Chardonnay y el Sauvignon Blanc, orientados fundamentalmente a los mercados de Estados Unidos y Europa.

La modernización del valle se inicia en la década de los 90, a partir de las primeras plantaciones en 1982, con 20 ha. que marcaron el inicio de la actividad y hoy día, su expansión alcanza 5.680 ha. (SAG 2008), con la presencia de bodegas que se destacan en el paisaje rural por su sofisticada arquitectura y el diseño ornamental de su entorno, las cuales forman parte de la llamada Ruta del Vino en el contexto del Enoturismo vinculado a la vitivinicultura, constituyendo uno de los circuitos recurrentes ofrecidos a los turistas de los cruceros marítimos que arriban cada año al puerto de Valparaíso.
Sin embargo, a pesar de sus potencialidades, la vitivinicultura del valle de Casablanca presenta ciertas vulnerabilidades que podrían ralentizar el desarrollo de la actividad a futuro o bien afectar incluso las actuales plantaciones, lo cual deriva de factores de tipo hidrográfico y climático que restringen la disponibilidad de riego, así como del incremento de la competencia vitivinícola de eventuales nuevas regiones productoras en el sur del país, derivado del cambio climático. Considerando estas limitaciones y los efectos espaciales, económicos y sociales producidos a la fecha por el desarrollo de la vitivinicultura y la modernización del Valle de Casablanca, se abre la interrogante respecto a las posibilidades reales de sustentabilidad de esta actividad en la Comuna de Casablanca, materia que se inserta en el Proyecto IPGH-OEA GEO 2.1.2.3.4, del cual deriva el presente artículo.

\section{MATERIALES Y MÉTODOS}

El artículo se circunscribe en el marco del proyecto IPGH-OEA titulado " Expansión de la Vitivinicultura y su relación con el Desarrollo Local en Casablanca (Chile) y Mendoza (Argentina)", el cual ha sido desarrollado en forma conjunta con académicos del Departamento de Geografía de la Universidad Nacional de Cuyo de Mendoza.

El área de estudio en Chile fue seleccionada por su nivel de especialización en el rubro, constituyendo uno de los valles más dinámicos y modernizados de la provincia de Valparaíso, destacando especialmente en la producción de cepas blancas y especialmente de la variedad chardonnay que constituye su producto estrella y le otorga identidad al valle.

El objetivo central del presente artículo es identificar los factores que conforman las ventajas comparativas y competitivas del valle de Casablanca para la producción de vinos finos de exportación, así como aquellos factores que representan una seria 
limitante para la expansión y sustentabilidad futura de esta actividad económica que orienta actualmente la modernización agrícola del valle.

Con este propósito, la modernización del valle de Casablanca se analizó considerando las siguientes variables: Cambios en la estructura productiva comunal, Estructura actual de la superficie vitícola, Sistemas de regadío y Localización de Agroindustrias del rubro. Las potencialidades vitivinícolas a su vez, fueron dimensionadas a partir de sus Ventajas Comparativas fundamentalmente de carácter agroclimático, las cuales fueron relacionadas con los requerimientos técnicos de producción de la especie, considerando una zonificación espacial al respecto, en tanto las Ventajas Competitivas se asocian a las vías de accesibilidad y a la existencia de bodegas o agroindustrias procesadoras. Finalmente, para el análisis de la vulnerabilidad de la actividad vitivinícola en el valle, se consideraron las limitantes en hidrografía, la infraestructura de riego y los sistemas de regadío imperantes, así como las eventuales amenazas derivadas del cambio climático y de la competencia de otras regiones productoras.

Las fuentes de información fueron de tipo bibliográfico, estadístico y cartográfico, complementado con entrevistas a informantes claves y actividades de terreno en el área de estudio.

\section{Marco Teórico-Conceptual}

\section{Globalización y Modernización productiva.}

La orientación actual de la economía mundial está definida por el proceso de Globalización, el cual puede ser caracterizado como una creciente gravitación de los procesos económicos, sociales y culturales de carácter mundial sobre aquéllos de índole nacional y regional, produciendo un redimensionamiento de las relaciones entre los Estados y la Sociedad en la medida que los espacios nacionales son transnacionaliza- dos generando nuevas identidades y estructuras de poder (DELGADILLO 2003).

El proceso de globalización está asociado a la búsqueda de nuevas formas de acumulación que operan a escala mundial y se traduce en nuevas estrategias técnico-económicas en el ámbito de la gestión, la producción y la comercialización de los productos con el objetivo de optimizar la productividad del capital, aunque sus repercusiones no ocurren solo en la esfera de lo económico, sino que se extienden al campo de lo social y cultural, modificando los patrones de consumo y comportamiento en general de la población (BERNAL-MEZA 2000).

La globalización proyecta una nueva visión acerca de los modos de aprehender el espacio que articula distintas escalas espaciales y temporales y donde conceptos como accesibilidad, conectividad e integración de territorios pasan a ser ejes directrices en la nueva lógica del capital operando a diferentes escalas. A escala global, los mercados mundiales sustituyen a los mercados nacionales como instrumentos de orientación de la producción; a escala regional se valorizan las ventajas comparativas y competitivas de los lugares y a escala local, se concreta la acción de los agentes económicos a través de la inversión productiva y la creación / destrucción de empleos (QUITARRÁ 1999).

El impacto de la globalización en las áreas rurales del tercer mundo ha significado revalorizar la importancia de la dotación de recursos naturales (clima, suelo, agua) y con ello la expansión de las actividades primarias relacionadas, como la fruticultura y silvicultura, junto con la agroindustria en el sector secundario, y en este contexto, la modernización de dichas actividades es una condición necesaria para insertar dichos productos en los mercados internacionales (RIFFO \& RETAMALES 2007).

La modernización como proceso de diferenciación estructural y funcional según el autor original de esta teoría antropológica 
(SMALSEN 1963, en LONG 1977), se define como un proceso por el cual unidades sociales más especializadas y más autónomas son establecidas. La modernización rural ocurriría a través de la innovación tecnológica y la aplicación del conocimiento científico; de la comercialización de los productos, (lo que caracteriza el paso de la agricultura de subsistencia a una comercial, acompañado de la especialización productiva a objeto de generar economías de escala); de la utilización del trabajo asalariado; de la industrialización de la agricultura a objeto de agregar valor y reducir la perecibilidad de los productos y finalmente, de la urbanización del campo, con cambios en la dimensión ecológica de las aldeas rurales hacia características propias de los núcleos urbanos.

Sin embargo, no todas las actividades y lugares son modernizados al mismo tiempo, coexistiendo por tanto economías tradicionales junto con aquéllas que han innovado al respecto y regiones o áreas ganadoras o perdedoras, según su capacidad para incorporarse al nuevo modelo de desarrollo imperante-lo cual es explicitado en la nueva Teoría del Dualismo Intra-Rural o de fragmentación rural (SILI 2002) -que rompe con la unidad del mundo rural, generando diferentes modelos productivos que responden a lógicas distintas de funcionamiento.

\section{Conceptos de Terroir y Denominación de Origen}

El concepto de terroir fue acuñado por los franceses (traducido a "terruño" por los españoles), para definir los lugares que le otorgan identidad a los vinos, originando un producto con características particulares que es determinado por el tipo de suelo, clima, topografía y la acción humana, en este caso, del enólogo.

La OIV (Organización Internacional de la Viña y el Vino 1999), define el terroir como una zona delimitada de tierras con denominación de origen, cuya naturaleza, configuración geográfica y clima permiten a los vitivinicultores elaborar productos específicos. Para VANDOR (2003), citado por BARAHONA (2005), el terroir es una entidad espacial y temporal que presenta características homogéneas del pedopaisaje y clima, asociado a un nivel de organización espacial y a un período dado, dentro de un territorio marcado por una historia social y de elecciones culturales. Según RIVELLA (1996), el terroir es el patrimonio exclusivo de cada zona de producción constituyendo un conjunto de condiciones únicas, siendo la conjunción de la planta, la tierra y la naturaleza con el hombre la que genera la diversidad de los vinos y les da su razón de ser.

Como progresivamente se elaboran vinos más parecidos y la difusión de las técnicas vitícolas y enológicas y el recurso de plantar variedades internacionales aumenta el riesgo de estandarizar la producción de vino en el mundo, surge la inquietud comercial para diferenciar la producción bajo el concepto de terroir, para identificar aquéllos con una personalidad marcada en aromas y sabores que aluden al terreno donde se cultiva la vid, en una evidente conexión entre el lugar geográfico en cuestión y el producto vino allí producido.

La influencia del terroir para los efectos de cultivar la vid, consiste en cualidades favorables de un lugar respecto al clima (como la temperaturas diurnas y nocturnas, insolación, amplitud térmica, pluviosidad, riesgo de heladas), el suelo (como drenaje) y topografía (relieve, altura respecto al nivel del mar). La diferencia entre un terroir y otro se expresa en el sabor y textura de los vinos, los cuales pueden aparecer distintos aunque sean derivados de la misma cepa, lo que le otorga exclusividad a las áreas de origen, favoreciendo al lugar con una identidad al respecto, lo que es destacado en el proceso de comercialización y de ahí su importancia en la producción de vinos finos.

Con el objeto de resguardar territorialmente las áreas productivas, se aplica en Chile 
el concepto de Denominación de Origen de los vinos en el año 1994 mediante Decreto Ley $\mathrm{N}^{\circ} 464$ de la Ley 18.455, modificada por la Ley 19.283 del Ministerio de Agricultura, que estableció la Zonificación Vitícola según criterio definido por las cuencas y valles existentes en el país, cuyas condiciones de clima, suelo, variedad de vides y prácticas culturales y enológicas sean homogéneas. Según dicha normativa, se establecieron 5 Regiones Vitivinícolas, subdivididas en 13 Subregiones, las que a su vez comprenden 7 Zonas que abarcan 44 áreas Vitícolas, con lo cual se delimitan las Denominaciones de Origen del país. En esta zonificación, el valle de Casablanca constituye una Subregión que junto con el Valle del Aconcagua forman parte de la Región Vitivinícola del Aconcagua (SAG 1996).

Según esta zonificación, los vinos podrán indicar en sus etiquetas la expresión "Embotellado de Origen", cuando al menos el $75 \%$ del vino en cuestión ha sido producido con uvas provenientes del lugar geográfico indicado.

\section{Antecedentes del Área de Estudio}

La comuna de Casablanca se ubica administrativamente en la provincia de Valparaíso, en la región homónima. Con una superficie de $952.5 \mathrm{~km} 2$, registra una población total de 21.874 habitantes (INSTITUTO NACIONAL DE ESTADISTICA, INE 2002) y una densidad de $23 \mathrm{hab} / \mathrm{km} 2$. La población rural es relativamente alta $(30,5 \%)$ en relación al promedio nacional de 2002 (13,5\%), la cual se encuentra concentrada en caseríos y aldeas o dispersa en el espacio rural, en tanto la población urbana $(69,5 \%)$ se concentra principalmente en la ciudad capital de Casablanca. La comuna presenta una excelente accesibilidad definida por la Ruta 68 que la conecta con Santiago (a $80 \mathrm{kms}$. aprox.) y con Valparaíso (a $40 \mathrm{~km}$ ), en tanto la Ruta K-90 la conecta con la ciudad de Algarrobo y el puerto de San Antonio (Fig. 1).

El Valle de Casablanca se ubica en la vertiente occidental de la Cordillera de la Costa, a 240 m.s.n.m. prolongándose hacia las planicies litorales donde se ubican los bal-

Fig.1: "ACCESIBILIDAD COMUNA DE CASABLANCA"

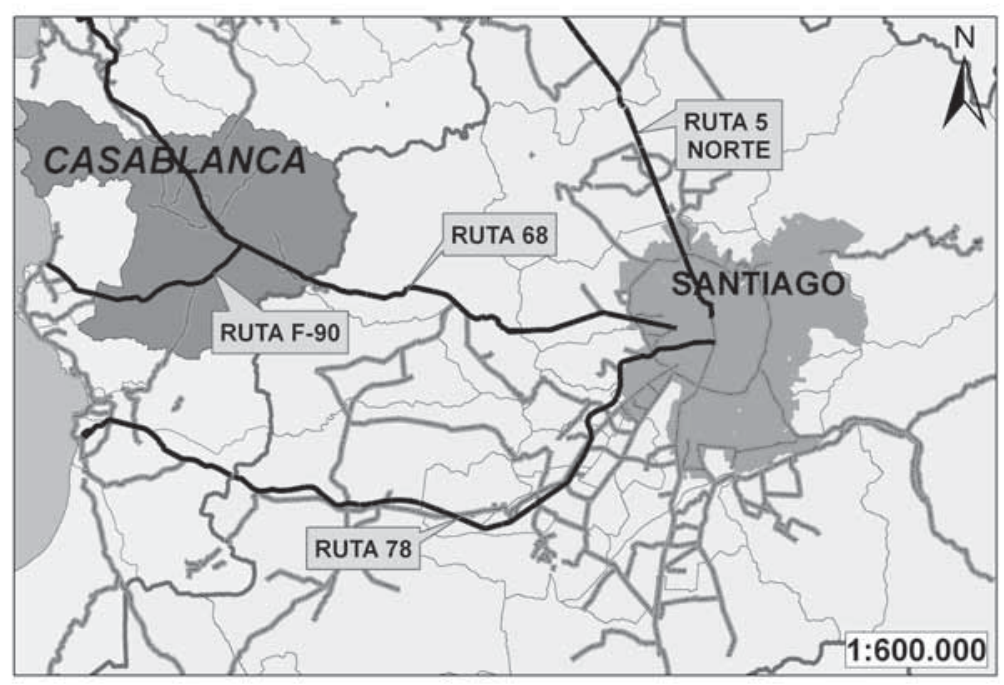

Fuente: Atlas Geográfico V Región. 
Fig.2: "CUENCAS Y SUBCUENCAS - COMUNA DE CASABLANCA"

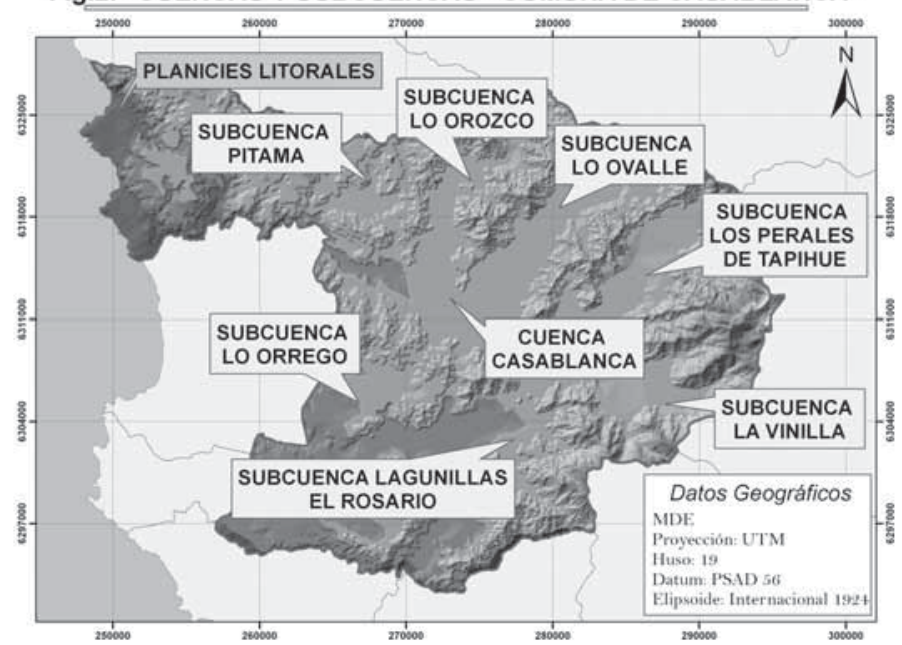

Fuente: Troncoso, 2006.

Fig.3: "RED HIDROGRÁFICA - COMUNA DE CASABLANCA"

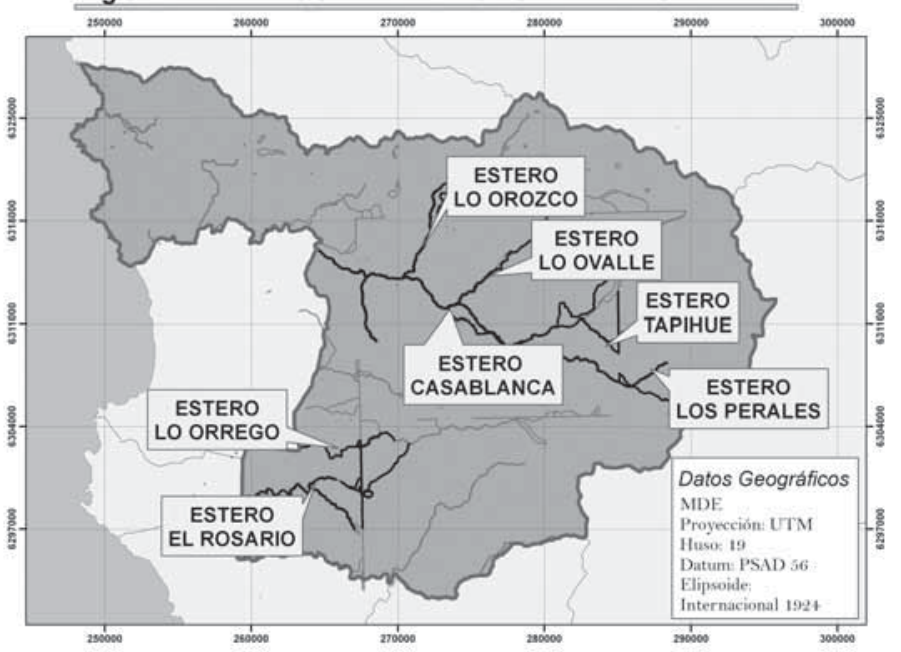

Fuente: Troncoso (2006).

nearios de Tunquén y Quintay. Geomorfológicamente, la comuna está compuesta por la gran cuenca Casablanca-Vinilla y las subcuencas Los Perales de Tapihue, Lo Ovalle, Lo Orozco, Pitama, Lo Orrego, Lagunillas y El Rosario (Fig. 2).

La gran cuenca es recorrida por el Estero Casablanca que se desplaza en sentido S.E - N.O desde sus nacientes en la Cordillera de la Costa con sus tributarios como los es- teros Los Perales, Tapihue, Lo Ovalle y Lo Orozco por el norte (Fig. 3).

Dada su ubicación geográfica, el valle carece de cursos de agua principal como ríos que puedan facilitar la construcción de embalses adecuados para el regadío, existiendo cinco de muy baja capacidad de almacenamiento (35 millones de $\mathrm{m}^{3}$ ) y de gran antigüedad (década del 30), por lo que la fuente principal de riego actual son los pozos de 
agua subterránea, cuyo abastecimiento depende en gran parte de las precipitaciones (Comisión Nacional de Riego 2003).

El clima es templado cálido con lluvias invernales y una estación seca prolongada de 7- 8 meses (promedio $483 \mathrm{mms}$. anuales) y su cercanía a la costa le permite el paso de la acción marina a través de vientos y la nubosidad típica de las mañanas, quedando despejados solo 180 días al año, contribuyendo con ello a mitigar la aridez climática, reduciendo la amplitud térmica, la evapotranspiración y el déficit hídrico. Los veranos secos y calurosos presentan temperaturas hasta $30^{\circ} \mathrm{C} \mathrm{y} \mathrm{los} \mathrm{inviernos} \mathrm{húmedos}$ temperaturas menores de $5^{\circ} \mathrm{C}$, llegando incluso a $0^{\circ} \mathrm{C}$. La temperatura media anual es de $13,6^{\circ} \mathrm{C}$ y la oscilación térmica anual es de $9^{\circ} \mathrm{C}$, siendo distintivo en Casablanca el extenso periodo libre de heladas $(10 \mathrm{me}-$ ses), las cuales se presentan generalmente en Julio y Agosto (Dirección Meteorológica de Chile 2002).

\section{RESULTADOS}

\section{Potencialidades de la vitivinicultura en el Valle de Casablanca}

Surgido en 1982 como Valle vitivinícola emergente, Casablanca fue el primero en la provincia y región de Valparaíso que adquirió fama mundial, con el vino chardonnay que constituye su "producto estrella", al que siguieron posteriormente otros valles, como el de San Antonio y Leyda.

Hasta la década de los 80, Casablanca era una comuna de agricultura tradicional donde predominaban los cultivos de cereales y chacras junto con la ganadería lechera. En 1982, el empresario Pablo Morandé inspirado en lo que observó en el valle de Napa en California, plantó las primeras 20 ha. de viñas (Chardonnay, Sauvignon Blanc y Riesling), siendo por tanto, un pionero en visualizar las potencialidades del valle para las cepas blancas en especial, lo cual fue imitado posteriormente por otros. En 1989 había solo 90 ha. plantadas, pero a partir de los 90' la situación cambió sustantivamente, iniciándose una reconversión productiva con el boom de plantaciones en el valle alcanzando en la actualidad 5.680 ha. plantadas (SAG 2008), dentro de un proceso que no ha culminado y que en la última década se ha caracterizado básicamente por la ocupación de laderas, en el contexto de una expansión vertical de la frontera agrícola en el Valle, lo cual le otorga un sello distintivo y atractivo al paisaje, visible desde la carretera principal (Ruta 68) que divide la comuna en sentido norte-sur.

Considerando las potencialidades del valle que le otorgan ventajas comparativas para la producción de cepas blancas, hay que hacer referencia a su ubicación geográfica a 240 m.s.n.m, en la vertiente occidental de la Cordillera de la Costa, la cual destaca por sus bajas cumbres lo que permite la influencia marítima del Océano Pacífico, como las brisas y neblina matinal, las cuales tienen una importancia significativa en la forma en que maduran las uvas y en el manejo que se debe tener en los viñedos.

Como el valle se extiende en un sentido EsteOeste, se generan dos sectores diferenciados con el camino a Algarrobo como frontera: el área occidental, conocida como "bajo Casablanca", que favorece la producción de vinos blancos por ser más húmedo y frío que el área oriental o "alto Casablanca", de naturaleza más cálida y seca por su mayor alejamiento del sector costero, todo lo cual potencia el desarrollo de cepas tintas, las cuales maduran más rápidamente que las blancas.

La tabla 1 muestra que la zona de "bajo Casablanca", presenta valores inferiores respecto a temperaturas mínimas, amplitud térmica, suma térmica y pluviometría, en tanto la zona de "alto Casablanca" lo hace con temperaturas máximas (aunque levemente), y días sin heladas (prácticamente todo el año) y muy significativamente respecto a horas-neblina. 
Tabla I. Zonificación Agroclimática del VALLE de CaSABlanca

\begin{tabular}{|c|c|c|}
\hline $\begin{array}{l}\text { VARIABLES } \\
\text { CLIMÁTICAS }\end{array}$ & $\begin{array}{c}\text { BAJO CASABLANCA } \\
(\mathrm{W})\end{array}$ & $\begin{array}{c}\text { ALTO CASABLANCA } \\
(\mathrm{E})\end{array}$ \\
\hline Tㅇ Máxima (promedio ${ }^{\circ} \mathrm{C}$ ) & 22.70 & 22.50 \\
\hline To Mínima (promedio ${ }^{\circ} \mathrm{C}$ ) & 4.80 & 6.60 \\
\hline Amplitud Térmica (promedio ${ }^{\circ} \mathrm{C}$ ) & 12.80 & 13.80 \\
\hline Suma Térmica (grados días) & 1.166 & 1.387 \\
\hline Horas- Neblina (año) & 3.601 & 24 \\
\hline Días sin Heladas (año) & 324 & 356 \\
\hline Pluviometría (mm. año) & 538 & 788 \\
\hline
\end{tabular}

Fuente: Villaseca \& Álvarez (2002)

La amplitud térmica influye en el aroma de los varietales y en la obtención del color de los vinos, mientras que la suma térmica, que se expresa en grados-días como diferencia entre una constante base de $10^{\circ} \mathrm{C}$ y el promedio de temperaturas diarias, influye a su vez en el desarrollo vegetativo de la especie. Entre los requerimientos climáticos de la vid son aceptables rangos entre 900 y 2.200 grados-días, por lo que las dos zonas presentan condiciones apropiadas al respecto. Lo mismo ocurre con los días sin heladas, ya que la especie requiere de 180 días y más sin la presencia de este problema, por cuanto la zona de "bajo Casablanca" presenta 324 días libres al año y la de "alto Casablanca" 356, valores muy superiores al umbral señalado, lo cual constituye una ventaja comparativa para el valle en general.

Técnicamente, las heladas ocurren en noches despejadas y tranquilas; las nubes, las neblinas o los vientos atrapan el calor acumulado en el suelo y en la piel de las plantas durante el día y en ausencia de estos factores, el calor se escapa y el aire se enfría, provocando heladas cuyo principal efecto es el de quemar las plantas, fenómeno que los productores combaten con diversos métodos, como el uso de aspersores que sustituyen al antiguo sistema de los aparatos de combustión (TAPIA 2004).

Como el aire frío es más denso que el caliente, los mayores daños ocurren en los sectores planos o bajos, donde el aire frío cae por su propio peso mientras que en las laderas, por su mayor altitud, el problema no se produce, lo cual le otorga una ventaja a estas áreas y explica la expansión vertical de la frontera agrícola en el valle a través de la habilitación de nuevas tierras productivas, lo cual es evidente en el sector norte de la comuna en torno a las subcuencas de Perales de Tapihue y Lo Ovalle preferentemente.

Uno de los aspectos en que sí se observan diferencias significativas al interior del valle es en lo relativo a las horas-neblina, lo cual es un efecto topoclimático vinculado al descenso de la temperatura bajo cero grados, lo que influye en el proceso de maduración de la uva, siendo más lenta en las áreas de mayor incidencia del fenómeno, y en este sentido, se observa que el sector poniente o "bajo Casablanca" presenta valores más altos por su mayor cercanía al Pacífico, que el sector oriente o " alto Casablanca", donde el índice es mínimo, aunque la especie puede desarrollarse dentro de un amplio rango de 20- 4000 horas-neblina. La neblina que viene del Pacífico, se desplaza desde la madrugada hasta el medio día cubriendo el valle con un gran manto de humedad, lo que obliga a los viticultores a tomar precauciones en el manejo del follaje para que los racimos estén en contacto con el sol y reciban una buena aireación y evitar al mismo tiempo que se retarde demasiado la madu- 
rez de la uva y surjan como consecuencia enfermedades producidas por hongos.

En términos generales, SANTIBÁÑEZ (1989) señala que el clima influye en un $90 \%$ en la conformación de un terroir, por cuanto incide fuertemente en el proceso de brotación, floración y maduración de la vid, y en este sentido, el valle de Casablanca por sus rasgos climáticos derivados de su situación geográfica en la cordillera de la Costa, constituye un área favorable para la producción de vinos blancos, como el Chardonnay, Sauvignon Blanc y Riesling y los vinos tintos de madurez corta, como el Pinot Noir y también el Merlot, lo cual ha marcado la especialización del valle.

Por su parte, su localización espacial con respecto a vías principales de transporte, constituye una de las ventajas competitivas del Valle de Casablanca; en efecto, el acceso expedito desde la ruta 68 le permite conectarse directamente hacia el poniente con el principal puerto del país (Valparaíso), como hacia el oriente con la capital nacional de Santiago. A su vez, la ruta F-90 le permite conectarse con el Puerto de San Antonio que constituye el principal puerto frutícola de exportación, de manera que la comuna de Casablanca configura un centro de gravedad entre los nodos de Santiago, Valparaíso y San Antonio.

\section{Desarrollo de la vitivinicultura en el Valle de Casablanca}

La expansión de la vitivinicultura y en especial de la viticultura comunal, se expresó inicialmente a partir del proceso de reconversión productiva, por el cual los rubros de cereales y frutales han sido gradualmente desplazados por los viñedos, los que junto con las empastadas y la silvicultura constituyen los sectores más dinámicos de la producción primaria, como se muestra en la tabla 2.

La tabla 2 muestra además de la reconversión señalada la ampliación del espacio productivo del valle en el período considerado, con la incorporación de 5.688 há. (53.5\%), situación que se explica fundamentalmente por la utilización de laderas viabilizado por la técnica del riego por goteo. Como se señaló anteriormente, la plantación de viñedos a mayor altitud presenta una ventaja comparativa con respecto a las tierras bajas, por cuanto enfrentan menor riesgo de heladas por una parte y de producción de hongos por otra, lo cual se expresa finalmente en menores costos de producción, una vez

Tabla 2. Cambios en la estructura productiva comunal (i990-200i)

\begin{tabular}{|c|c|c|c|c|c|c|}
\hline 1990 (ha.) & $\%$ & Uso del suelo & 2001 (ha.) & $\%$ & Variación (ha.) & $\%$ \\
\hline 2.928 & 27.4 & Cereales & 641 & 3.9 & -2.287 & -78.1 \\
\hline 3.972 & 37.2 & Plantaciones Forestales & 5.460 & 33.4 & 1.488 & 37.5 \\
\hline 1.272 & 11.9 & Empastadas & 5.700 & 34.8 & 4.428 & 348.1 \\
\hline 1.239 & 11.6 & Viñas & 3.652 & 22.3 & 2.413 & 198.8 \\
\hline 612 & 5.7 & Frutales & 316 & 1.9 & -296 & -48.4 \\
\hline 376 & 3.5 & Chacras & 189 & 1.2 & -187 & -49.7 \\
\hline 264 & 2.4 & Hortalizas & 398 & 2.4 & 134 & 50.8 \\
\hline 13 & 0.1 & Otros & 8 & 0.0 & -5 & -38.5 \\
\hline 10.676 & 99.8 & Total & 16.364 & 99.9 & 5.688 & 53.3 \\
\hline
\end{tabular}

Fuente: Troncoso (2006). 
Tabla 3: Estructura de la superficie vitícola del valle de Casablanca

\begin{tabular}{|l|l|l|r|r|r|r|}
\hline 2006 (ha.) & \multicolumn{1}{|c|}{ CEPAS } & 2008 (ha.) & \multicolumn{2}{c|}{$\%$} & \multicolumn{2}{|c|}{ VARIACIÓN } \\
\hline & & & & & (ha.) & \multicolumn{1}{c|}{$\%$} \\
\hline 1.773 & 46.3 & Chardonnay (blanca) & 2.269 & 39.9 & 496 & 28.0 \\
\hline 756 & 19.7 & Sauvignon Blanc (blanca) & 1.932 & 34.0 & 1.176 & 155.5 \\
\hline 475 & 12.4 & Pinot Noir (tinta) & 710 & 12.5 & 235 & 49.5 \\
\hline 434 & 11.3 & Merlot (tinta) & 397 & 7.0 & -37 & -8.5 \\
\hline 3.438 & 89.8 & Sub Total & 5.308 & 93.5 & 1.870 & 54.4 \\
\hline 390 & 10.2 & Otras & 372 & 6.5 & -18 & -4.6 \\
\hline 3.828 & 100 & Total & 5.680 & 100 & 1.852 & 48.4 \\
\hline
\end{tabular}

Fuente: Elaboración propia en base a información SAG.

Tabla 4. Participación de las cepas principales del valle de Casablanca

\begin{tabular}{|c|c|l|c|c|}
\hline \multicolumn{2}{|c|}{2006} & \multicolumn{1}{c|}{ CEPAS } & \multicolumn{2}{c|}{2008} \\
\hline Ha. & $\%$ & & Ha. & $\%$ \\
\hline 2.529 & 73,6 & Blancas & 4.201 & 73,9 \\
\hline 909 & 26,4 & Tintas & 1.107 & 19,5 \\
\hline 3.438 & 100 & Sub total & 5.308 & 100 \\
\hline
\end{tabular}

Fuente: Elaboración propia en base a información SAG.

superado el de la habilitación de suelos en laderas y la implementación del riego por goteo.

Como se observa en la tabla 3 , se distinguen cuatro cepas dominantes dentro de la estructura productiva vitícola del Valle de $\mathrm{Ca}$ sablanca, tanto en el año 2006 como 2008, representadas por dos blancas y dos tintas, situación que se acentúa en el 2008 donde aumenta la participación de ellas, desde el $89.8 \%$ al $93.5 \%$.

Sin embargo, en este grupo marcan presencia dominante las cepas blancas, situación que se mantiene en el 2008, subiendo levemente su participación del $73.6 \%$ al $73.9 \%$ (tabla 4), lo cual estaría demostrando la especialización productiva del valle de acuerdo a sus ventajas comparativas de tipo climático, fundamentalmente. No obstante ello, la tabla 3 muestra que entre las cepas blancas se observa un cambio en el ritmo de expansión relativa, donde la cepa Chardonnay crece más lentamente en el 2008 (28\%) con respecto al Sauvignon Blanc (155.5\%), reduciendo su participación total del $46.3 \%$ al $39.9 \%$ aunque continua siendo dominante, constituyendo aún el "producto estrella" de Casablanca, seguido de cerca por el Sauvignon Blanc con $34.0 \%$. Finalmente, en términos generales, se constata una expansión de $48.4 \%$ de la superficie plantada entre los dos años considerados, con 1.842 nuevas hectáreas, lo cual demuestra que esta actividad sigue siendo muy atractiva para los inversionistas del sector.

A nivel nacional, destacan las plantaciones de la cepa Cabernet Sauvignon que constituye la principal variedad plantada en el país con el 37,1 \% de participación, en tanto las cepas blancas Chardonnay y Sauvignon Blanc alcanzan en conjunto solo el $22.9 \%$ del total con 23.983 ha. (tabla 5). Dentro de este contexto, la comuna de Casablanca representa el $17.5 \%$ con 4.201 ha. de estas dos variedades (tabla 6), muy diferente del 
Tabla 5. Distribución de las PRincipales CEPAS EN LAS PLANTACiONES DE VIDES VINÍFERAS DEL PAÍS (2008).

\begin{tabular}{|l|c|c|}
\hline \multicolumn{1}{|c|}{ CEPAS } & HECTÁREAS & $\%$ \\
\hline Cabernet Sauvignon & 38.806 & 37.1 \\
\hline Chardonnay & 12.739 & 12.2 \\
\hline Sauvignon Blanc & 11.244 & 10.7 \\
\hline Merlot & 9.656 & 9.2 \\
\hline Carmenere & 8.249 & 7.9 \\
\hline Syrah & 5.391 & 5.1 \\
\hline Pinot Noir & 2.598 & 2.5 \\
\hline Otras & 16.034 & 15.3 \\
\hline Total & 104.717 & 100 \\
\hline
\end{tabular}

Fuente: Servicio Agrícola Ganadero (2008)

Tabla 6. Participación de la comuna en las Plantaciones de Variedades BLANCAS DEL PAÍS.

\begin{tabular}{|c|c|l|c|c|}
\hline $\begin{array}{c}\text { PAIS } \\
(\mathrm{Ha})\end{array}$ & $\%$ & \multicolumn{1}{|c|}{ CEPAS } & COMUNA (Ha) & \% DE PARTICIPACIÓN \\
\hline 12.739 & 12.2 & Chardonnay & 2.269 & 17.8 \\
\hline 11.244 & 10.7 & Sauvignon Blanc & 1.932 & 17.2 \\
\hline 23.983 & 22.9 & Sub Total & 4.201 & 17.5 \\
\hline
\end{tabular}

Fuente: Elaboración propia en base a información SAG

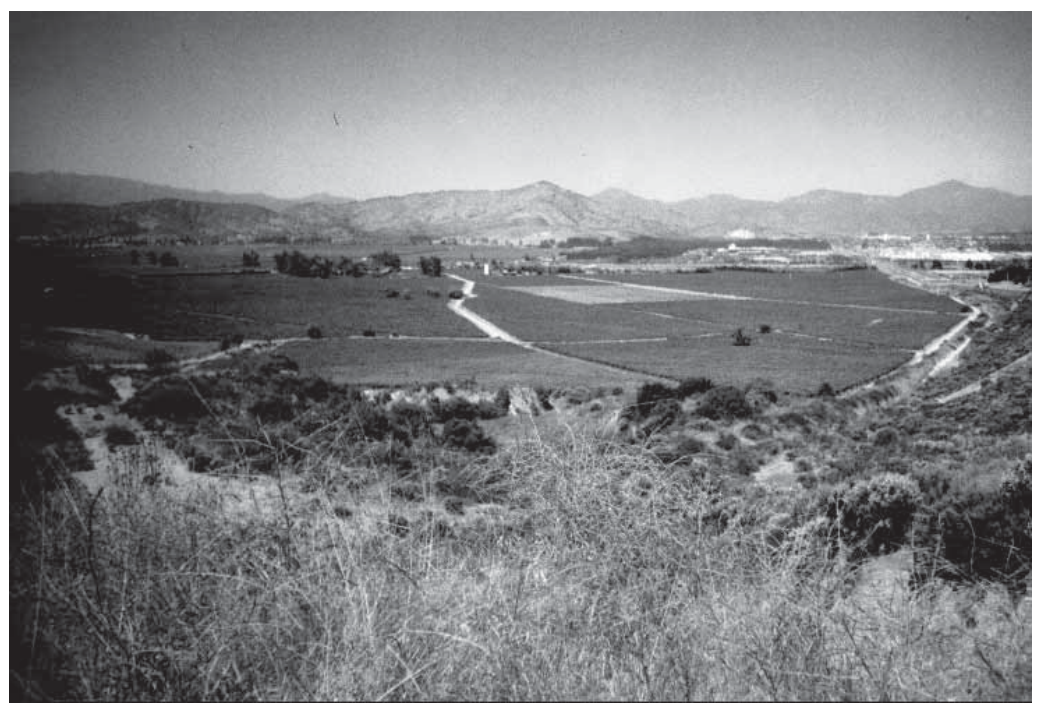

Fig. 4. Panorámica del valle de Casablanca. Fuente: Trabajo de terreno 2008 
$73.9 \%$ que estas mismas representan a escala intra-comunal, lo cual evidencia el nivel de especialización de la comuna de Casablanca.

Por su parte, la expansión de la superficie plantada con viñedos en general, ha sido también estimulada por la localización de empresas vitivinícolas en el valle, las cuales constituyen las llamadas "Bodegas Boutiques," las que se ubican próximas a la ruta 68 destacándose en el paisaje rural por su particular diseño arquitectónico y su entorno paisajístico, las cuales no solo conforman puntos de venta de sus productos, sino que además cuentan con restaurantes que reciben visitas nacionales e internacionales, formando parte de la Ruta del Vino de Casablanca (Fig. 5 y 6 ).

Las bodegas (ocho en total) son tanto de origen nacional como extranjero y disponen de sus propios viñedos, además de la compra de materia prima a terceros. La superficie plantada de estas empresas alcanza a 1.062 há. en el 2006, representando el $27.7 \%$ de la superficie total con viñedos de entonces (3.838 ha), en tanto la producción sumó ese año 410.500 cajas, destacando al respecto las bodegas Veramonte con 200.000 cajas (48,7\%), Indómita con 100.000 (24.4\%), William Cole con 50.000 (12.2\%) y Casas del Bosque con 40.000 cajas ( 9.7\%). La producción de vinos finos es exportada sobre el $80 \%$, principalmente a Estados Unidos y Europa occidental (Inglaterra, Irlanda, Suecia) y en menor medida a América Latina (Brasil, México) y Asia (Japón, China) (TRONCOSO 2006).

En general, la presencia de estas empresas vitivinícolas en el valle han comandado el proceso de modernización agrícola mediante la innovación tecnológica, tanto en viñedos como en la industria y la promoción de los vinos con denominación de origen del

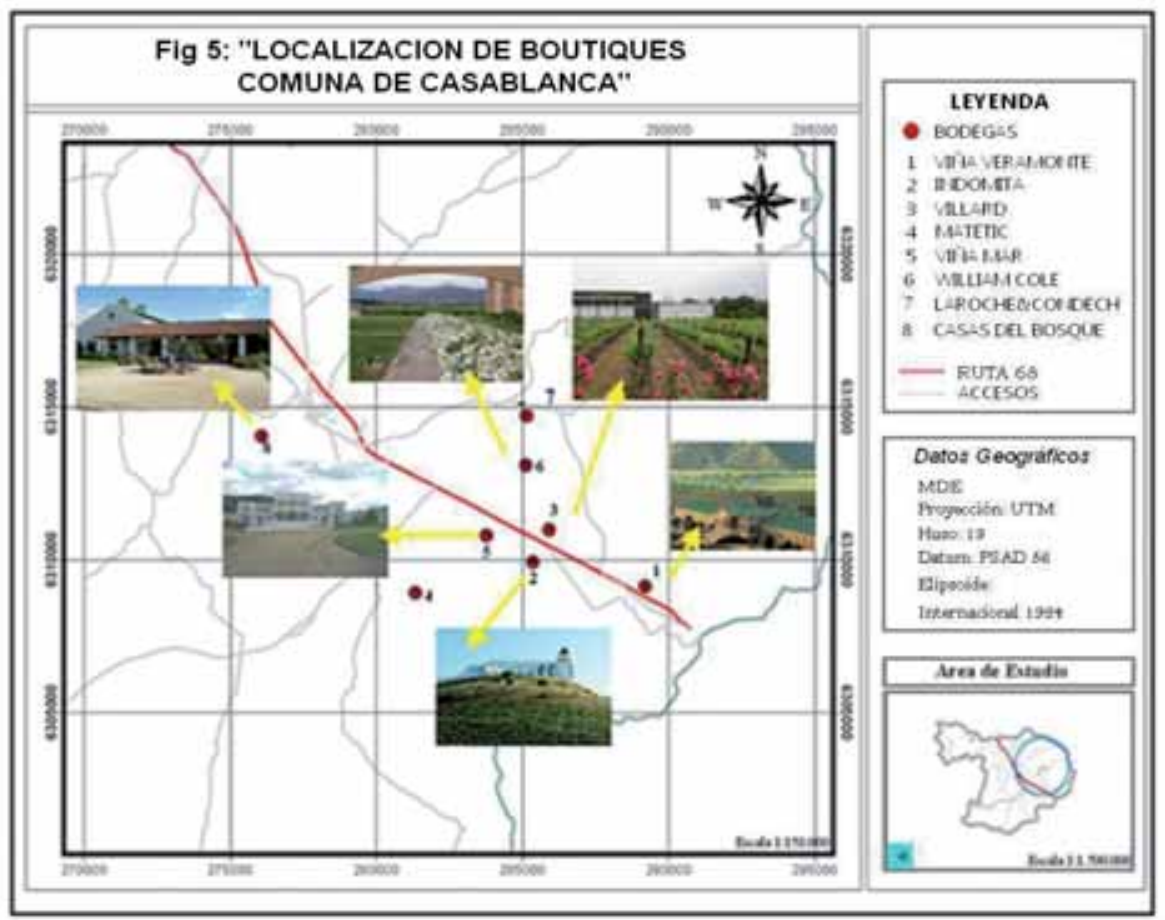

Fuente: Troncoso (2006). Trabajo de terreno durante 2008. 


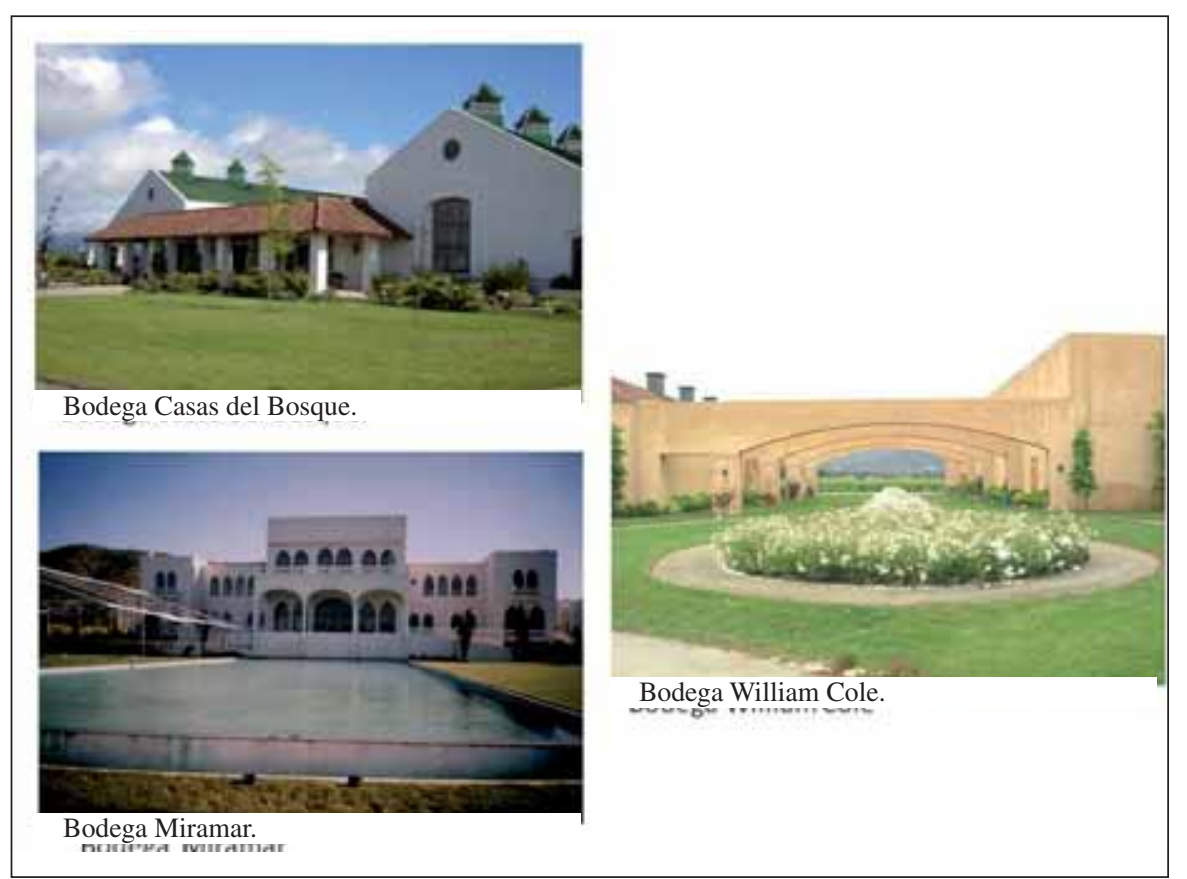

Fig. 6. Bodegas boutique del valle de Casablanca. Fuente: Trabajo de terreno durante 2008.

Valle de Casablanca, estimulando la reconversión productiva y la expansión vertical de la frontera agrícola con la ocupación creciente de las laderas que circunscriben las subcuencas de la comuna.

\section{Vulnerabilidad de la Vitivinicultura de Casablanca}

Los factores que constituyen limitantes actuales o potenciales de la actividad en el Valle de Casablanca son fundamentalmente de dos tipos: uno de carácter endógeno que se relaciona con la disponibilidad de Infraestructura de Riego y dos de carácter exógeno referidos a la demanda y precios de los mercados internacionales, junto con la amenaza de la competencia de regiones productoras alternativas derivado del cambio climático.

\section{Las limitantes de la Infraestructura de Riego}

Considerando su ubicación espacial en el flanco occidental de la Cordillera de la Costa, el Valle de Casablanca carece de cursos de agua principales - como son los ríos siendo el estero de Casablanca su principal sistema hidrográfico, el cual se desplaza en sentido Este-Oeste y se alimenta fundamentalmente de las precipitaciones que se presentan en las estaciones de otoño e invierno (483 mm. anuales), ya que la baja altitud de la cordillera de la Costa no permite la producción y acumulación de nieve y su aporte a los cursos de agua superficial en primavera.

No obstante esta limitante, el valle dispone de cinco embalses construidos en la década del 30, los que se abastecen fundamentalmente de las lluvias por lo que su aporte se restringe en el período estival, cuando jus- 
Tabla 7: Embalses del valle de Casablanca

\begin{tabular}{|c|c|c|c|c|}
\hline Embalses & $\begin{array}{c}\text { Capacidad } \\
\text { (millones m3) }\end{array}$ & $\begin{array}{c}\text { Superficie } \\
\text { Beneficiada (ha) }\end{array}$ & $\begin{array}{c}\text { Año de } \\
\text { Construcción }\end{array}$ & Administración \\
\hline Lo Ovalle & 13.5 & 1.2 & 1932 & Privada \\
\hline Tapihue & 11.6 & 800.0 & 1932 & Privada \\
\hline Lo Orozco & 5.5 & 800.0 & 1932 & Privada \\
\hline Purísima & 2.4 & 400.0 & 1931 & Privada \\
\hline Pitama & 2.0 & 400.0 & 1932 & Privada \\
\hline Total & 35.0 & 2.401 .2 & & \\
\hline
\end{tabular}

Fuente: Comisión Nacional de Riego (2003)

Tabla 8. Fuentes de agua y Superficie Regada del valle de Casablanca.

\begin{tabular}{|l|c|c|}
\hline \multicolumn{1}{|c|}{ Tipo de Fuente } & Superficie Regada (hectáreas) & $\%$ \\
\hline Pozos (119) & 5.254 & 62.5 \\
\hline Embalses (5) & 2.401 & 29.7 \\
\hline Vertientes & 197 & 2.4 \\
\hline Canales & 160 & 2.0 \\
\hline Norias & 63 & 0.8 \\
\hline Total & 8.075 & 100 \\
\hline
\end{tabular}

Fuente: Troncoso (2006)

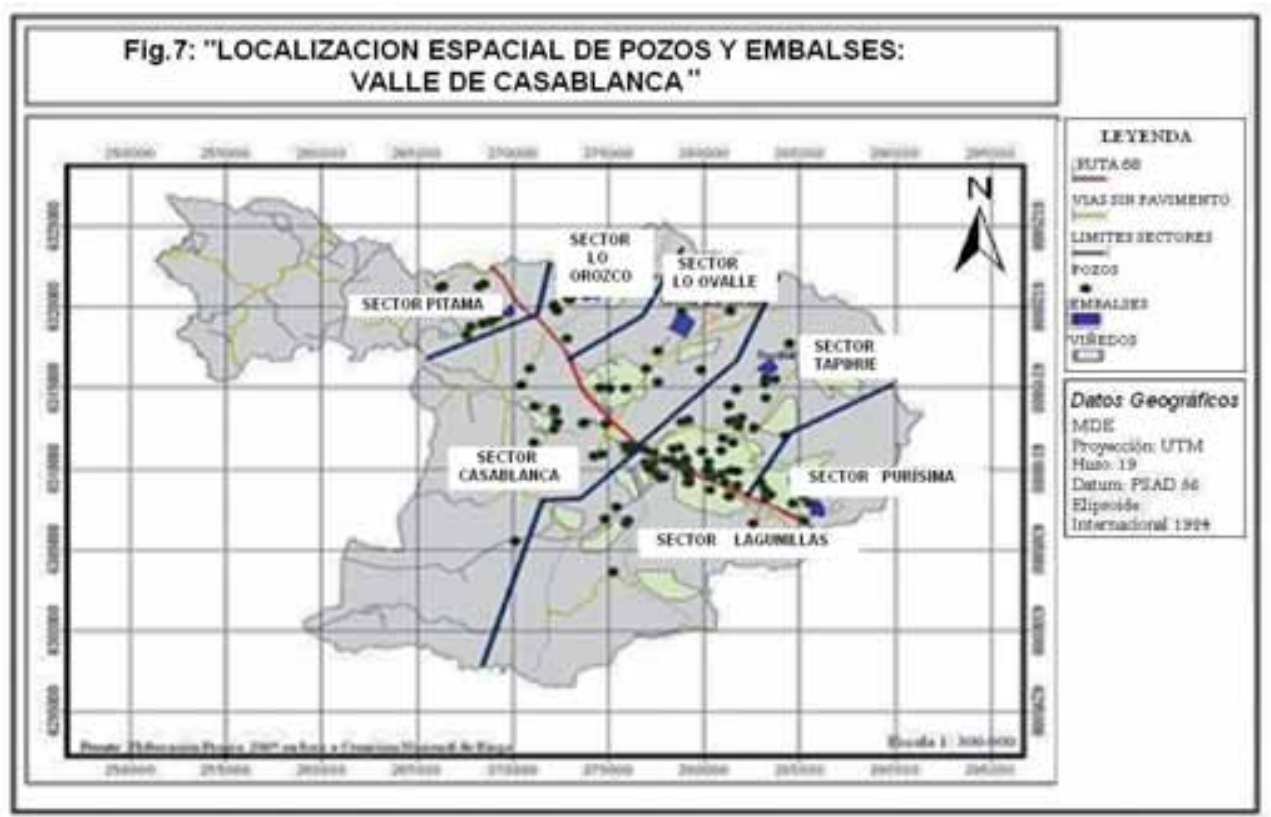

Fuente: Troncoso (2006) 
tamente se requiere del recurso para el regadío. Como muestra la tabla 7, la capacidad de los embalses es muy baja ( 35 millones de $\mathrm{m}^{3}$ en total), siendo proyectada su cobertura para 2.401 há, (lo cual resulta restringida dada su actual obsolescencia), que representa solo el $29,7 \%$ de la superficie regada del valle.

Dada la limitante de agua superficial para el regadío en el Valle de Casablanca, la napa de agua subterránea emerge como principal fuente de abastecimiento mediante 119 pozos explotados, los cuales riegan el 62,5\% de la superficie productiva total, concentrándose espacialmente en dos sectores del valle (La Vinilla y Los Perales) situados al oriente de la Comuna (tabla 8 y Fig. 7).

Otra limitante referida al riego son los sistemas imperantes en el valle, (tabla 9), donde se constata que el sistema de Inundación es el más utilizado (48.8\%), seguido del Surco $(31,2 \%)$ y en tercer lugar el riego por Goteo, con solo el $13.4 \%$ de la superficie regada, el cual predomina espacialmente en los sectores Vinilla Abajo y Los Perales. Ésto significa que siendo escasa el agua disponible, ésta es también mal utilizada por cuanto se pierde gran parte del recurso en los sistemas de Inundación y Surco. Considerando que la modernización agrícola se caracteriza por la optimización de los sistemas de regadío que se logra con el riego por goteo, podría señalarse entonces, que en este sentido la modernización del valle de Casablanca es incompleta, con solo 810 ha. cubiertas con este sistema, orientándose preferentemente hacia los sectores de producción en laderas.

\section{Variaciones de la demanda, Competen- cia alternativa y Cambio climático}

Por otra parte, las eventuales variaciones de la demanda internacional, con la fluctuación de los mercados en términos de la inestabilidad de la demanda y de los precios, de sustitución con otros licores, de competencia de regiones/países alternativos y de variación en el valor del dólar, constituyen amenazas para la actividad vitivinícola en el Valle de Casablanca.

De estos factores, la competencia creciente de Argentina como país exportador representa el mayor riesgo, aunque el producto estrella de Mendoza como gran zona productiva es la cepa tinta Malbec, pero con introducción reciente de cepas blancas, lo cual implica necesariamente fortalecer las

Tabla 9. Sistemas de Riego y Superficie Regada (HA.) En el valle de Casablanca.

\begin{tabular}{|l|r|r|r|r|r|r|r|}
\hline Localidad & Inundación & Surco & California & Aspersión & Goteo & TOTAL & $\%$ \\
\hline La Vinilla Abajo & 43 & 363 & 48 & 309 & 641 & 1404 & 23,0 \\
\hline Los Perales & 1.107 & 460 & 0 & 22 & 51 & 1640 & 27,0 \\
\hline Tranque Perales & 291 & 0 & 0 & 0 & 0 & 291 & 4,8 \\
\hline Los Perales Arriba & 7 & 0 & 0 & 0 & 0 & 7 & 0,1 \\
\hline Casablanca & 684 & 212 & 0 & 0 & 0 & 896 & 15,0 \\
\hline Lo Ovalle Abajo & 424 & 425 & 17 & 0 & 97 & 963 & 16,0 \\
\hline Lo Orozco Abajo & 202 & 69 & 0 & 0 & 0 & 271 & 4.5 \\
\hline Lo Orozco Arriba & 55 & 28 & 0 & 0 & 0 & 83 & 1.4 \\
\hline Las Dichas & 73 & 0 & 0 & 0 & 0 & 73 & 1.2 \\
\hline Lo Ovalle Arriba & 52 & 320 & 0 & 0 & 21 & 393 & 6.5 \\
\hline La Vinilla Arriba & 0 & 0 & 0 & 0 & 0 & 0 & 0 \\
\hline Total & 2.938 & 1.877 & 65 & 331 & 810 & 6.021 & 100 \\
\hline$\%$ & 48.8 & 31.2 & 1.1 & 5.5 & 13.4 & 100 & \\
\hline
\end{tabular}

Fuente: Elaboración Propia en base a I. Municipalidad de Casablanca (2001). 
campañas de marketing a partir de la asociatividad de los actores involucrados en Casablanca (gobierno local, empresas vinícolas y viñateros), para competir no sólo con Argentina, sino también con los otros productores exportadores del hemisferio sur, como son Australia, Nueva Zelanda y Sudáfrica.

En el contexto de regiones productoras alternativas de cepas blancas, como el Chardonnay y el Sauvignon Blanc, es pertinente colocar el escenario derivado de un eventual cambio climático que favorezca el desarrollo de otras áreas competidoras en el país, con o sin el perjuicio de estas producciones en Casablanca por anulación de sus actuales ventajas comparativas al respecto, tanto por incremento de las temperaturas como por reducción de las precipitaciones en el valle.

Un incremento de las precipitaciones incide en una mayor oferta de agua y en mayores rendimientos, aunque ello depende de la oportunidad de las lluvias en relación al ciclo vegetativo de las plantas. Respecto a la temperatura, un incremento implica acelerar el crecimiento de las plantas alcanzando la madurez en menor tiempo, lo que en el caso de las vides viníferas implicaría un aumento de la acidez, favoreciendo con ello el desarrollo de las variedades tintas.

Aunque la agricultura tiene la capacidad de adaptarse a los cambios climáticos graduales, es muy vulnerable a los eventos extremos que pueden afectar seriamente las plantaciones, como son las sequías y las inundaciones, lo cual es importante considerar en el análisis de la sensibilidad de respuesta de las plantas. En el caso de las sequías, las plantas sufren estrés hídrico cuando el agua de la cual disponen, (sea por reservas subterráneas, precipitaciones o acumulaciones de nieve en las altas cumbres), es inferior a la evapotranspiración, la cual es inducida en su mayor parte por la temperatura, y por lo tanto, un escenario climático de fuerte aumento de temperatura con disminución de las precipitaciones provocaría un mayor es- trés hídrico en las plantas, sin que ello signifique una respuesta de tipo lineal en todos los casos (BOULANGER 2007).

Según SANTIBÁÑEZ (2007), en Chile las temperaturas máximas han disminuido y las mínimas han aumentado en los últimos años, lo que implica un incremento de las temperaturas promedio y una reducción de la oscilación térmica, lo que en el caso de las vides viníferas influye en el aroma de los varietales y el color de los vinos. A su vez, los otoños están siendo cada vez más secos en Chile y las lluvias más aleatorias y asociadas a eventos más intensos, lo que en productos templados como la vid implicaría mayores requerimientos de riego para neutralizar los efectos de sequías.

El estudio de SANTIBÁÑEZ (2007) mostró que se produciría un calentamiento significativo hasta la IX región, lo que traería como consecuencia un desplazamiento de la agricultura hacia el sur, incluyendo las regiones de Los Ríos y Los Lagos, que por su clima templado y mayor pluviosidad pueden ser una alternativa viable para el desarrollo de las variedades blancas, lo cual obviamente requeriría del proceso modernizador pertinente para la vitivinicultura -la que en la actualidad se extiende desde la región de Coquimbo a la del Bio Bio-, las cuales podrían incorporarse como zonas productoras competidoras a Casablanca.

Según estudio de la CEPAL y el Gobierno de Chile (2009) sobre "La Economía del cambio climático en Chile", las proyecciones de los especialistas estiman un aumento significativo de las temperaturas en 4 grados implicaría una reducción de hasta $30 \%$ de las precipitaciones desde la Región de Valparaíso hasta Los Lagos hacia fines de siglo, situación que para el valle de Casablanca sería muy negativo, dado que las principales fuentes de agua derivan de las lluvias que alimentan los esteros y los pozos de agua subterránea, de los cuales depende a su vez el regadío para la vitivinicultura. 
En el seminario "El cambio climático y sus efectos en el agua" organizado por la Cámara Chileno-Británica de Comercio (2009), se planteó que en los últimos 30 años de este siglo habrá entre 12 y 14 sequías, cuyos principales impactos van a estar asociados a la disminución de los recursos hídricos y de la productividad, lo que obligará a hacer un uso más eficiente de las aguas subterráneas dado que el alza de las temperaturas aumentará la evaporación de las fuentes superficiales de agua, y que aquellas áreas donde la agricultura está funcionando en el umbral del recurso, no podrán superar el cambio climático. Como resultado, el territorio de las vides productoras de vino emigrará al sur del país, debido a que la falta de agua en el centro reducirá los rendimientos y encarecerá los costos de producción haciendo menos rentable la actividad.

\section{DISCUSIÓN}

El valle de Casablanca forma parte del espacio vitivinícola de Chile, cuya modernización y especialización productiva se inicia en la década de los 90 a partir de un proceso gradual de reconversión productiva desde la agricultura tradicional, proceso que aún no ha concluido si se consideran las tierras actualmente ocupadas con cereales y chacras y las laderas disponibles para ser incorporadas a la producción.

Considerando los resultados obtenidos del estudio, se evidencia que la especialización vitivinícola del valle se sustenta básicamente en sus ventajas comparativas para la actividad, complementado con las ventajas competitivas de accesibilidad y presencia de agroindustrias procesadoras o bodegas boutiques. En lo primero, su ubicación espacial en los contrafuertes de la cordillera de la costa, contribuye a generar condiciones agroclimáticas favorables para el desarrollo de cepas blancas, donde la neblina costera cumple un rol fundamental al propiciar la lenta maduración que caracteriza a estas cepas, como son el chardonnay y el sauvignon blanc, las cuales encuentran un nicho productivo especialmente en el sector occidental del valle, denominado "bajo Casablanca", en tanto hacia el interior - sector de "alto Casablanca"- las condiciones climáticas con menor influencia oceánica permiten el desarrollo de cepas tintas de madurez corta, como son el pinot noir y el merlot.

Las cepas blancas marcan la especialización vitivinícola del valle, por cuanto en el 2008 representaban el $73,9 \%$ de las plantaciones frente al $19.5 \%$ de las cepas tintas. Considerando esta relación desigual, la competencia vitícola se da al interior de las cepas blancas, al menos por ahora y sin cambio climático de por medio, y en este sentido, el chardonnay que hasta el 2006 constituía el producto estrella que le otorgaba identidad al valle con el $46.3 \%$ de las plantaciones, en el 2008 reduce su participación al 39.9\%, en tanto su competidor sauvignon blanc avanza desde el $19.7 \%$ al $34 \%$, lo cual implica que hay factores que estarían modificando el comportamiento de los productores, aunque el valle en general siga siendo un nicho favorable para las cepas blancas.

Sin embargo, la modernización del valle y las perspectivas de expansión futura de las plantaciones, presenta una limitante determinada fundamentalmente por la escasez del recurso hídrico derivado de la inexistencia de cursos de agua principal, como son los ríos, lo que a su vez limita la construcción de nuevas obras de infraestructura de riego como los embalses, unido al hecho de que los existentes a la fecha (5), están obsoletos y disponen de muy baja capacidad de almacenamiento ( 35 millones de m3). Ello explica que solo el $29.7 \%$ de la superficie regada del valle provenga de los embalses y que sean los pozos de agua subterránea (119) la fuente principal del riego $(62.5 \%)$.

A ello se agrega el hecho que los sistemas de riego imperantes en el valle sean principalmente por inundación (48.8\%) y surco $(31.2 \%)$ y solo el $13.4 \%$ por goteo, lo que 
estaría indicando que el proceso de modernización en este aspecto es incompleto y que se está derrochando un recurso que es escaso, tanto por la falta de cursos de agua superficial como por escasez de precipitaciones (483 mm/anuales), constituyendo el agua de riego por lo tanto, un recurso limitado y limitante al mismo tiempo.

Esta situación se hace más evidente, si se considera que la ampliación de la superficie plantada se ha manifestado en la última década a través de la incorporación de laderasproceso de expansión vertical de la frontera agrícola- lo cual resulta factible solo con la disponibilidad de riego por goteo, por lo que podría suponerse que en la medida que este proceso continúe en el valle, el sistema por goteo se incrementará correlativamente.

De lo anteriormente señalado, se deduce que las ventajas comparativas- que son naturales- tienen una doble lectura en el valle de Casablanca. Por una parte, el clima determina condiciones muy favorables para la especialización productiva en viñas de cepas blancas, lo cual ha sido aprovechado en el sector poniente del valle, en tanto las precipitaciones de clima mediterráneo, ésto es, escasas en primavera-verano, hacen imperativo el regadío artificial, para lo cual se requiere de ríos- inexistentes en el valle, constituyendo un factor limitante para el desarrollo de la actividad, lo cual requiere ser compensado con un uso más racional y eficiente del agua de riego.

Finalmente, otro factor limitante a futuro, sería el eventual cambio climático en el valle, que se podría manifestar a través de una reducción de las precipitaciones - lo que agudizaría el problema del regadío- y el incremento de las temperaturas que podría afectar las ventajas agroclimáticas de las cepas blancas, las cuales podrían encontrar nichos más favorables en el sur del país, como en las regiones de Los Ríos y Los Lagos, en tanto en el valle de Casablanca es posible que las cepas tintas surjan con mayor potencialidad dentro de este nuevo escenario climático, siempre que los inversionistas mantengan su interés en este valle.

\section{CONCLUSIONES}

En el contexto de la globalización, las regiones de los países en desarrollo han logrado insertarse a través de la exportación de productos que presentan ventajas comparativas y competitivas en los mercados mundiales. En este escenario, el valle de Casablanca destaca por la producción y exportación de vinos finos, y preferentemente de cepas blancas.

El nivel de especialización en viñedos se inicia con el reconocimiento de ventajas comparativas fundamentalmente climáticas para el desarrollo de la actividad, donde la ubicación geográfica en el flanco occidental de la cordillera de la Costa y la influencia de las brisas marinas del Pacífico y la neblina costera resultan ser determinantes para la producción de cepas blancas, con la definición de áreas que constituyen el terroir para la producción del Chardonnay que constituye su "producto estrella" que le otorga la identidad al valle de Casablanca. A ello se agrega la ventaja competitiva de su excelente nivel de accesibilidad, tanto a Santiago como a los principales puertos del país, como son Valparaíso y San Antonio, que constituyen ejes directrices de la nueva lógica del capital operando a diferentes escalas, esto es, a nivel local o comunal para la producción y a nivel mundial para la comercialización, por cuanto son los mercados internacionales los instrumentos de orientación de la producción de los vinos finos con denominación de origen.

Las ventajas comparativas y competitivas señaladas han estimulado la localización espacial en el Valle de ocho empresas vitivinícolas y con ello la reconversión productiva desde la agricultura tradicional y posteriormente la expansión de la frontera agrícola con la producción en laderas, lo que marca la actual fisonomía del paisaje rural de 
la comuna. Sin embargo, la reconversión productiva no es total, lo que implica que en el valle de Casablanca co-existen actividades tradicionales, como la producción de cereales y chacras, y actividades modernas como la vitivinicultura, las que responden a distintos modelos de producción y lógicas de funcionamiento, dando paso con ello a un dualismo de carácter intra-rural.

La vitivinicultura se ha desarrollado desde la década de los 90, con la creciente expansión de la superficie plantada con viñedos, la producción de vinos finos y la exportación a Estados Unidos y Europa preferentemente. Sin embargo, la expansión de la actividad no es indefinida en el tiempo y el espacio, por cuanto surgen ciertas limitantes que representan niveles de vulnerabilidad que eventualmente pueden llegar a frenar el desarrollo de la vitivinicultura en el valle e incluso afectar su consolidación como actividad y este riesgo deriva de las deficiencias en su infraestructura de riego, dado que carece de cursos de agua superior y los embalses existentes disponen de escasa capacidad y se encuentran en situación de obsolescencia dada su antigüedad (década del 30).

Por otra parte, el proceso de modernización del valle es incompleto en términos tecnológicos, por cuanto predominan aún los sistemas de riego tradicional, como lo son el de inundación y surco, que se traducen en la pérdida del recurso agua, en tanto el sistema moderno de riego por goteo que caracteriza a las áreas más modernizadas del país, presenta escaso desarrollo aún y en este sentido el agua constituiría una fuerte limitante para la expansión futura de las plantaciones especialmente en laderas.

La actividad depende también de factores externos no controlados por los productores, como son la demanda de los mercados internacionales, la competencia de los países exportadores alternativos, como Argentina, Australia y Nueva Zelanda en el hemisferio Sur y finalmente, de los eventuales cambios climáticos que podrían derivar del calentamiento global, lo cual podría afectar la producción de cepas blancas del valle de Casablanca desplazándose hacia el sur con la incorporación de nuevas regiones productoras, como La Araucanía, Los Ríos y Los Lagos, que con su clima templado lluvioso se podrían adscribir a la vitivinicultura de exportación, previo proceso de modernización.

Dicho escenario futuro implicaría que como efecto del calentamiento global, el valle de Casablanca podría llegar a conformar un área de producción de cepas tintas más que de cepas blancas, compitiendo con el resto del país al respecto, lo que podría significar a su vez, un eventual reemplazo de los actores vitivinícolas y con ello la generación de nuevas estructuras de poder e identidad, a partir de la pérdida de la denominación de origen, el terroir, para las cepas blancas.

Finalmente, esta vulnerabilidad demuestra que las actividades agrícolas e industriales derivadas, como la vitivinicultura, siguen siendo dependientes de factores naturales a pesar de los avances tecnológicos, a lo que se agregan los factores económicos y políticos no controlados por los actores económicos y ello abre la interrogante respecto a las perspectivas reales de desarrollo local para la Comuna de Casablanca a partir de la especialización en una actividad económica dominante, en este caso, la vitivinicultura, y del rol que a los gobiernos y a la población local les compete desempeñar para lograrlo.

\section{REFERENCIAS}

BARAHONA, A.M., 2004. ¿De qué hablamos cuando hablamos de Terroir? Revista de la Vendimia. Santiago, Chile. 3 pp.

BERNAL-MEZA, R. 2000. Sistema Mundial y MERCOSUR. Globalización, Regionalismo y Políticas Exteriores Comparadas. Editorial Nuevo Hacer. Buenos Aires, Argentina. $480 \mathrm{pp}$ 
BOULANGER, J.P., 2007. Impacto del clima en el sistema agrícola: Estrategias de Comunicación con los niveles de conducción y decisión. Selección de artículos de Le Monde diplomatique: La Agricultura ¿Otra víctima del cambio climático? Santiago, Chile. 33-48.

CÁMARA CHILENO-BRITANICA DE COMERCIO, 2009. El cambio Climático sus Efectos en el Agua. Seminario Facultad de Economía y Negocios. Universidad de Chile. Santiago, Chile.

CEPAL, 2009. La Economía del Cambio Climático en Chile. Naciones Unidas. Santiago, Chile. 88 pp.

COMISIÓN NACIONAL DE RIEGO, 2003. Cartografía Digital de Pozos y Embalses. Ministerio de Obras Públicas. Santiago, Chile.

DELGADILLO, J., 2003. Reestructuración económica territorial en el marco de la Globalización. Universidad Autónoma de México. D.F. 12 pp.

DIRECCIÓN METEOROLÓGICA DE CHILE, 2002. Resumen de Temperaturas y Precipitaciones. Comuna de Casablanca. Dirección General de Aeronáutica Civil, Santiago, Chile.

INSTITUTO NACIONAL DE ESTADÍSTICAS, INE, 2002. XVII Censo Nacional de Población y VI de Vivienda. Santiago, Chile.

LONG, N., 1977. Introdução a Sociología do Desenvolvimento Rural. Zahar Editores (traducción). Río de Janeiro, Brasil. 237 pp.

QUITARRÁ, O., 1999. Globalización: Reflexiones desde la Geografía. Revista Reflexiones Geográficas. Río Cuarto, Argentina. 8:71-78

RIFFO, M. \& RETAMALES, C. 2007. Dinámica de cambios en el patrón de asentamiento de la población rural: Región del Maule. Revista Investigaciones Geográficas Universidad de Chile. Santiago, Chile. 39: 62-90.
RIVELLA, E., 1996. La Denominación de Origen como instrumento de penetración en los mercados. Seminario Internacional de Viticultura, Enología y Comercialización. Universidad Católica de Chile. Santiago, Chile.

SANTIBÁÑEZ, F; DÍAZ, F; GAETE, C., DANERI, S; \& D. DANERI, 1989. Agroclimatología y Zonificación de la región vitivinícola chilena: Bases para una Denominación de Origen de los vinos. Proyecto 074/ 84 del Fondo Nacional de Desarrollo Científico y Tecnológico. Santiago, Chile.

SANTIBÁÑNE, F., 2007. Los cambios climáticos globales ¿Qué le deparan a Chile? En.: Le Monde diplomatique: La Agricultura ¿Otra víctima del cambio climático? Santiago, Chile. 75-83.

\section{SERVICIO AGRÍCOLA Y GANADERO,}

SAG. 1996. Legislación Vitivinícola y Denominaciones de Origen. Ministerio de Agricultura. Santiago, Chile. 63 pp.

SERVICIO AGRÍCOLA Y GANADERO, SAG. 2008. Catastro Vitícola Nacional. Ministerio de Agricultura. Santiago, Chile. 17 pp.

SILI, M., 2002. Del Enfoque Agrario al Enfoque Rural. Ideas y Conceptos para construir una Nueva Ruralidad. En Revista Universitaria de Geografía. Universidad Nacional del Sur. Bahía Blanca, Argentina, pp. 71-90.

TAPIA, P., 2004. Descorchados. La Guía de Vinos. Editorial Planeta. Santiago, Chile. 289 pp.

TRONCOSO, R., 2006. Efectos socioespaciales derivados de la Vitivinicultura: Caso Comuna de Casablanca, V Región de Valparaíso. Memoria para optar al título de Geógrafo, Facultad de Arquitectura y Urbanismo. Universidad de Chile. Santiago, Chile. 118 pp Inédita

VILLASECA, S. \& ALVAREZ, M. (2002). Zonificación Agroclimática del Valle de Casablanca. Revista Tierra Adentro. Santiago, Chile. Mayo-Junio. 44: 26-2. 